\title{
Project ECHO: Filling the Gap in Pediatric Palliative Care Training in India
}

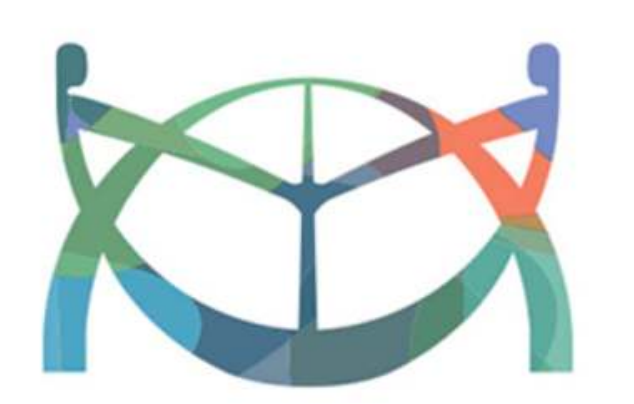

WOWORLDS

\section{Emily Evans ${ }^{1}$, Megan Doherty ${ }^{2,5}$, Spandana Rayala ${ }^{3,5}$, Gayatri Palat ${ }^{4,5}$}

1. University of Ottawa, Faculty of Medicine

3. Hyderabad Centre for Palliative Care

5. Two Worlds Cancer

2. Children's Hospital of Eastern Ontario

4. MNJ Institute of Oncology

4. MNJ Institu
Collaboration

\section{BACKGROUND}

- More than $98 \%$ of children who need palliative care live in low- or middle-income countries, where access to palliative care is very limited. ${ }^{1}$

- Education for healthcare professionals has been identified as a key initiative to improve access to pediatric palliative care services in India. ${ }^{2,3}$

- Project ECHO (Extension for Community Healthcare Outcomes) for Pediatric Palliative Care is an online educational program that was developed to provide specialist training for healthcare professionals in South Asia, allowing them to provide better palliative care services to seriously ill children in their communities.

- The one-year program was launched in March 2018 and uses webbased videoconference sessions to create a supportive global learning community.

- Regular 90-minute sessions were hosted every two weeks and included didactic teaching led by an international expert and an interactive case presentation focused on a particular topic within pediatric palliative care.
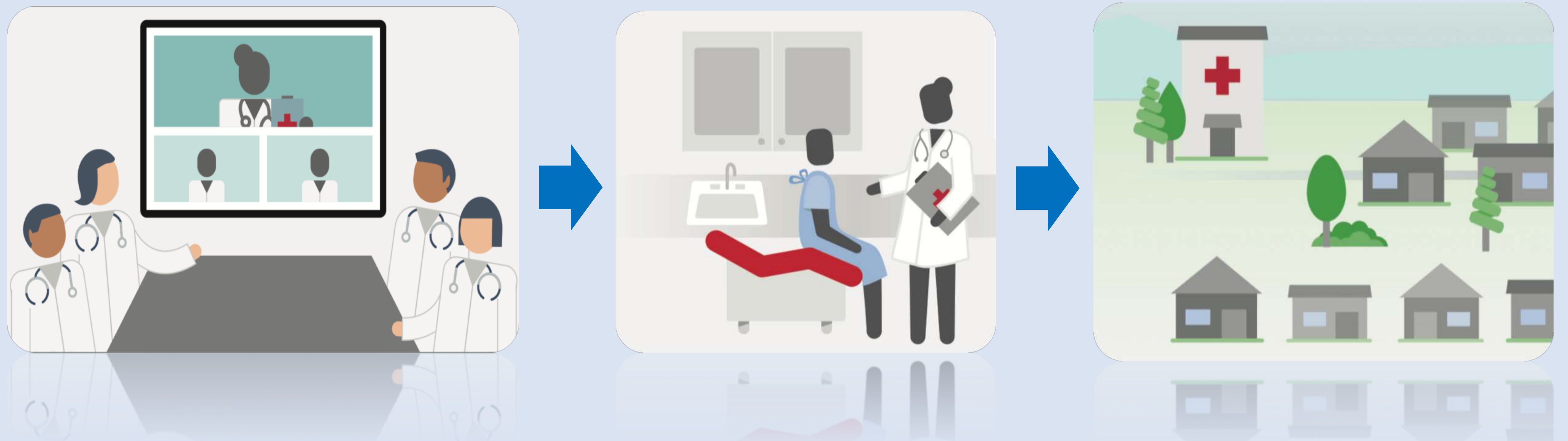

\section{OBJECTIVES}

- The goal of this study was to evaluate the extent of participation in the program, including the demographics of participants, distribution of participating health centres across South Asia, and session frameworks.

- An assessment of participant satisfaction with the program and its perceived strengths and weaknesses will provide feedback for the future implementation of similar educational programs.

\section{METHODS}

- Process outcomes, including the number of participants, their demographics, duration of each portion of the session, number of questions asked during discussions, and time during which participants and facilitators spoke, were recorded for each session.

- Participant satisfaction, perceived strengths and weaknesses, suggestions for improvement, and barriers preventing regular participation, were assessed using a paper survey.

\section{RESULTS}

- More than 150 different healthcare providers from 47 different centres across South Asia have participated in the program.

- Of participating centres, the majority were hospitals $(37 \%)$, NGOs $(28 \%)$ or community/homecare services $(22 \%)$.

- Most participants were physicians (47\%) or nurses (42\%).

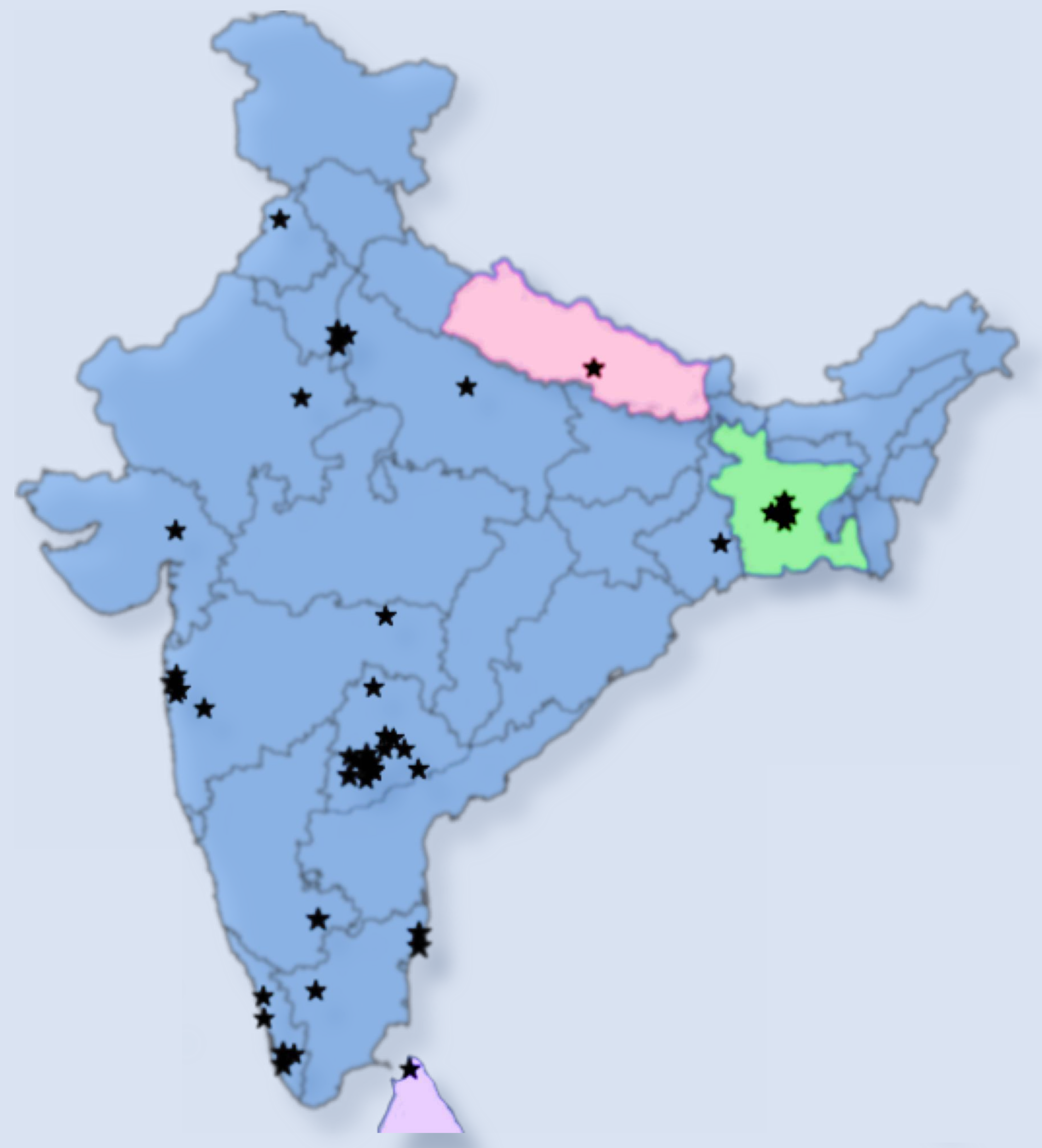

Map illustrating centres participating in Project ECHO PPC across India, Nepal, Bangladesh, and Sri Lanka.

- On average, 36 participants from 16 different centres attend each session.

- The majority of participants rated their experience with the ECHO program very positively.

- Highlighted strengths of the program include:

- Creating a multidisciplinary global participation

- Sharing experiences of participants during case discussions

- Learning resources that are provided after each session.

Poor internet network connectivity and limited access to technology were described as main weaknesses of the program.

\section{CONCLUSION}

- Project ECHO PPC is an innovative educational format for delivering pediatric palliative care education in resource limiting settings, which bridges the gap between experts and practitioners through an online community.

In its first year, Project ECHO PPC has received positive reviews by participants, with new participants continuing to join from across South Asia.

In the future, the ECHO team plans on expanding our program to target institutional teaching of medical trainees, nurses, and other healthcare professionals.

\section{REFERENCES}

Sasaki, Connor, S.R., Downing, J., Marston, J., 2017. Estimating the Global Need for Palliative Care for Children: A Cross-sectional Analysis. J. Pain Symptom Manage. 53, 171-177.

2. H., Bouesseau, M.C., Marston, J., Mori, R., 2017. A scoping review of palliative care for children in lowand middle-income countries. BMC Palliat. Care 16, 60.

3. McDermott, E., Selman, L., Wright, M., Clark, D., 2008. Hospice and Palliative Care Development in India: A Multimethod Review of Services and Experiences. J. Pain Symptom Manage. 35, 583-593. 